\title{
AUTHOR INDEX Volume 9 (2013)
}

Abd El-Samie, F. E., see

Hussein, G. A.

Abd El-Samie, F. E., see

Madkour, M. E.

Agrawal, A., see Rautaray, S. S.

Al-Hanafy, W., see Hussein, G. A.

Alshebeili, S. A., see

Hussein, G. A.

Arafat, H. A., see El-Said,

A. M.

Cacciola, M., Occhiuto, G.

\& Morabito, F. C.,

Artistic Complexity and

Saliency: Two Faces of

the Same Coin?

Cai, Y., see Yang, X.

Chen, C., see Han, L.

Chen, Y.-L., see Han, L.

Chen, Z., Wu, J. \& Chi, Z., A Mutation Particle Swarm Optimization Algorithm for Multilayer Perceptron Training with Applications

Cheng, Y., see Liu, H.

Chi, Z., see Chen, Z.

Dejey, see Sugi, T.

de Silva, C. N., see de Silva, C. W.

de Silva, C. W., see

Raman, S.

de Silva, C. W., Xiao, S., Li, M. \& de Silva, C. N., Sensory Signal Processing Issues in a Telemedicine System

Dessouky, M. I., see Hussein, G. A.
2 (2013) 1350013

3 \& 4 (2013) 1350018

3 \& 4 (2013) 1350017

1 (2013) 1350007

3 \& 4 (2013) 1350018

3 \& 4 (2013) 1350018

1 (2013) 1350006

2 (2013) 1350010

3 \& 4 (2013) 1350019

2 (2013) 1350009

2 (2013) 1350009

1 (2013) 1350003

1 (2013) 1250004

1 (2013) 1350003

1 (2013) 1350008

2 (2013) 1350013

1 (2013) 1350001

3 \& 4 (2013) 1350018
Dessouky, M. I., see Madkour, M. E.

3 \& 4 (2013) 1350017

Doyle, D., see Lieberman, M.

Eldesoky, A. I., see El-Said, A. M.

3 \& 4 (2013) 1350014

1 (2013) 1350006

Eldokany, I. M., see

Hussein, G. A.

3 \& 4 (2013) 1350018

El-Rabaie, E.-S. M., see

Hussein, G. A.

3 \& 4 (2013) 1350018

El-Said, A. M., Eldesoky,

A. I. \& Arafat, H. A., An Efficient Object Oriented Text Analysis (OOTA) Approach to Construct Static Structure with Dynamic Behavior

Feng, Y., see Tan, Z.

Gao, R., see Yang, J.

Han, L., Wu, X., Ou, Y., Chen, Y.-L., Chen, C. \&

Xu, Y., Household Service Robot with Cellphone

Interface

2 (2013) 1350009

Han-Lin, H., see Jian-Jun, $\mathrm{T}$.

1 (2013) 1350005

Hussein, G. A., Nofal, M. A., Dessouky, M. I., Oraby, O. A., Al-Hanafy, W., Mohamed, A. E.-N. A., Eldokany, I. M., El-Rabaie, E.-S. M., Alshebeili, S. A. \& Abd El-Samie, F. E., Supporting Distress Signals Over Low Earth Orbit Mobile Satellite Systems for Emergency Information Acquisition 
Jian-Jun, T., Han-Lin, H. \&

Ping, X., Auxiliary

Adaptive Mechanism in

Chaos Synchronization:

Estimators for Lipschitz

Constant and Upper

Bound of Time-Delay's

Change Rate

Jing, W., A New Modified

Particle Swarm

Optimization for

Multilayer Perception

Training with

Applications

Kong, B., see Xu, Y.

Li, M., see de Silva, C. W.

Lieberman, M., Muresan,

R., Yang, S. X. \& Doyle,

D., Hilbert-Huang

Transform for Feature

Extraction of Temperature

Modulated MOS Sensors

Liu, H., Cheng, Y. \& Meng,

M. Q.-H., A Variational

Multiphase Level Set

Algorithm for Image

Segmentation Based on

Active Contour Model

Luo, M., see Tan, Z.

Madkour, M. E., Soliman, S. E., Dessouky, M. I. \& Abd El-Samie, F. E.,

Coding and Interleaving

Schemes for Information

Acquisition from Wireless

Sensor Networks

Meng, M. Q.-H., see

Liu, H.

Meng, M. Q.-H., see

Yang, J.

Mohamed, A. E.-N. A., see

Hussein, G. A.

Morabito, F. C., see

Cacciola, M.

Muresan, R., see

Lieberman, M.

Nandi, G. C., see Pandey, S. C.

Nofal, M. A., see Hussein, G. A.

Occhiuto, G., see Cacciola, M.
1 (2013) 1350005

1 (2013) 1350004

3 \& 4 (2013) 1350016

2 (2013) 1350013

3 \& 4 (2013) 1350014

1 (2013) 1250004

2 (2013) 1350012

3 \& 4 (2013) 1350017

1 (2013) 1250004

1 (2013) 1350002

3 \& 4 (2013) 1350018

2 (2013) 1350010

3 \& 4 (2013) 1350014

3 \& 4 (2013) 1350015

3 \& 4 (2013) 1350018

2 (2013) 1350010
Oraby, O. A., see Hussein, G. A.

3 \& 4 (2013) 1350018

Ou, Y., see Han, L.

Pandey, S. C. \& Nandi, G. C., Artificial Endocrine System: A New Paradigm of Knowledge Discovery

Ping, X., see Jian-Jun, T.

Purohit, G. N., see Sharma, S.

Rajesh, R. S., see Sugi, T.

Raman, S. \& de Silva, C.

W., Sensor-Fault

Tolerant Condition

Monitoring of an

Industrial Machine

Rautaray, S. S. \& Agrawal, A., Vision-Based Application-Adaptive Hand Gesture Recognition System

1 (2013) 1350007

Sadasivam, V., see

Sindhuja, A.

1 (2013) 1250005

Sharma, S. \& Purohit, G. N., A Novel Framework for Tracking Online Community Interaction in Social Network

3 \& 4 (2013) 1350015

1 (2013) 1350005

2 (2013) 1350011

1 (2013) 1350008

1 (2013) 1350001

Sindhuja, A. \& Sadasivam, V., Multi-Feature-Based Segmentation of Sonoelastographic Breast Images

1 (2013) 1250005

Soliman, S. E., see Madkour, M. E.

Song, X., see Tan, Z.

Sugi, T., Dejey \& Rajesh, R. S., Geometric Attack Resistant Robust Image Watermarking Scheme

Tan, Z., Luo, M., Feng, Y., Zhuang, X. \& Song, X., Research of Printing Quality Detection Based on Image Restoration

Tian, Q., see Xu, Y.

Tseng, C., see Yang, X.

Wei, H., see Xu, Y.

Wu, J., see Chen, Z.

Wu, X., see Han, L.

Xiao, S., see de Silva, C. W.
3 \& 4 (2013) 1350017

2 (2013) 1350012

1 (2013) 1350008

2 (2013) 1350012

3 \& 4 (2013) 1350016

3 \& 4 (2013) 1350019

3 \& 4 (2013) 1350016

1 (2013) 1350003

2 (2013) 1350009

2 (2013) 1350013 
Xu, Y., Kong, B., Wei, H. \& Tian, Q., Lane-Based Direction Marking Recognition Using $\mathrm{Hu}$ Moments

3 \& 4 (2013) 1350016

$\mathrm{Xu}, \mathrm{Y}$., see Han, L.

Yang, J., Gao, R. \& Meng, M. Q.-H., Nonlinear Control for $G A L$

Regulatory Network in S. cerevisiae
2 (2013) 1350009

1 (2013) 1350002
Yang, S. X., see Lieberman, M.

3 \& 4 (2013) 1350014

Yang, X., Cai, Y. \& Tseng, C., An Interactive Computer Framework for Learning Genetics

3 \& 4 (2013) 1350019

Zhang, H., A Fast

Palmprint Verification

System Based on Fractal Coding

1 (2013) 1250003

Zhuang, X., see Tan, Z.
2 (2013) 1350012 